\title{
Distance Dependence of Triplet Energy Transfer in Water and Organic Solvents: A QM/MD Study
}

\author{
Carles Curutchet ${ }^{* a}$ and Alexander A. Voityuk ${ }^{* b c}$
}

aDepartament de Fisicoquímica, Facultat de Farmàcia, Universitat de Barcelona, Av. Joan XXIII s/n, 08028 Barcelona, Spain

' Institució Catalana de Recerca i Estudis Avançats (ICREA), 08010 Barcelona, Spain

'Institut de Química Computacional and Departament de Química, Universitat de Girona, Campus Montilivi, 17071 Girona, Spain

*To whom correspondence should be addressed. E-mail: carles.curutchet@ub.edu and alexander.voityuk@icrea.cat 


\begin{abstract}
The possibility to optimize optoelectronic devices, such as organic light-emitting diodes or solar cells, by exploiting the special characteristics of triplet electronic states and their migration ability, is attracting increased attention. In this study, we analyze how an intervening solvent modifies the distance dependence of triplet electronic energy transfer (TEET) processes by combining molecular dynamics simulations with quantum chemical calculations of the transfer matrix elements using the Fragment Excitation Difference (FED) method. We determine the $\beta$ parameter characterizing the exponential distance decay of TEET rates in a stacked perylene dimer in water, chloroform and benzene solutions. Our results indicate that the solvent dependence of $\beta$ ( $\beta_{\text {vacuum }}=5.14$ $\AA^{-1}>\beta_{\text {water }}=3.77 \AA^{-1}>\beta_{\text {chloroform }}=3.61 \AA^{-1}>\beta_{\text {benzene }}=3.44 \AA^{-1}$ ) can be rationalized adopting the McConnell model of superexchange, where smaller triplet energy differences between the donor and the solvent lead to smaller $\beta$ constants. We also estimate the decay of hole transfer (HT) and excess electron transfer (EET) processes in the system using the Fragment Charge Difference (FCD) method, and find that $\beta_{\text {TEET }}$ can be reasonably well approximated by the sum of $\beta_{\mathrm{EET}}$ and $\beta_{\mathrm{HT}}$ constants.
\end{abstract}

KEYWORDS: Triplet excitation energy transfer, electron transfer, hole transfer, solvent effects, distance dependence, superexchange. 


\section{Introduction}

Triplet-triplet electronic energy transfer (TEET) is an important process in materials and life sciences, which can be viewed as a simultaneous transfer of two electrons of opposite spin. ${ }^{1}$ In photosynthesis, undesired chlorophyll triplet states are quenched through triplet transfer reactions in order to avoid the generation of harmful singlet oxygen. ${ }^{2}$ Recently, the study of TEET has attracted increased attention due to the many applications of the process in the context of organic optoelectronics. ${ }^{3-4}$ For instance, a detailed knowledge of the fate of triplet excitons is expected to improve the efficiency of organic light-emitting diodes (OLEDs), given that spin statistics predict a larger proportion of triplet excitons compared to singlets upon charge injection. ${ }^{5}$ Another attractive application consists in design of photovoltaic devices, either exploiting the long lifetime of triplet states, which can lead to increased exciton diffusion lengths, ${ }^{6}$ or by using photon up-conversion. ${ }^{7}$ In the last process, low-energy triplets are generated by fast intersystem crossing in a sensitizing layer, and then transferred through TEET to a photoactive layer where triplet-triplet annihilation generates higher energy singlet excitons. ${ }^{8}$

Despite these and other applications, TEET has been far less studied compared to singlet energy transfer or electron transfer reactions. Early studies by Closs and coworkers, however, already realized the close correspondence between TEET and electron transfer, ${ }^{9-10}$ as they observed that the electronic coupling matrix elements mediating TEET, hole transfer (HT) and excess electron transfer (EET) reactions for a series of bridged dyads are related via the following equation:

$$
\left|V_{\text {TEET }}\right| \approx C\left|V_{E E T}\right|\left|V_{H T}\right|
$$

where the constant $\mathrm{C}$ depends on the particular system under study. Some years later, the theoretical analysis of Scholes and co-workers showed that TEET orbital overlapdependent interactions are indeed mediated by charge-transfer configurations, rather than the Dexter exchange interaction, ${ }^{11}$ and derived approximate theoretical expression connecting the TEET coupling to HT and EET matrix elements, thus providing theoretical support for the relation in Eq. 1. ${ }^{12-13}$ The experiments by Closs and coworkers, in addition, revealed an exponential attenuation of TEET couplings with donor-acceptor separation consistent with the superexchange model originally developed by McConnell for electron transfer. ${ }^{14}$

Recent studies have address in detail the mechanisms of TEET in both donor-bridgeacceptor systems and $\pi$-stacked molecules. ${ }^{5,15}$ From a theoretical point of view, considerable effort has been directed toward the development of accurate methods to estimate electronic couplings, a key parameter that controls TEET. ${ }^{16-19}$ In such studies, the distance dependence of TEET rates, typically characterized by a decay parameter $\beta$, 
was analyzed both for inter and intramolecular transfers. In contrast to electron transfer, ${ }^{20-22}$ though, no detailed studies on the modulation of the TEET coupling by an intervening solvent have been presented. If one assumes that HT and EET exhibit very close exponential distance attenuation factors $\left(\beta_{\mathrm{HT}} \approx \beta_{\mathrm{EET}}\right)$, according to Eq. 1 the TEET decay parameter should follow the relation $\beta_{\mathrm{TEET}} / \beta_{\mathrm{EET}}=2$. However, the validity of this relation for solvent modulated TEET remains unclear. In particular, Mani and coworkers found that in covalently linked porphyrin-Rhodamine dyads $\beta_{\mathrm{TEET}} / \beta_{\mathrm{EET}}=1.3,{ }^{23}$ which significantly differs from the ratio $\beta_{\mathrm{TEET}} / \beta_{\mathrm{EET}}=2$.

In this work, we analyze in detail the distance dependence of electronic coupling values for triplet energy migration in a stacked model perylene diimide (PDI) dimer in water and two organic solvents of different polarity, and compare the results with the distance dependence for the related HT and EET reactions. We chose the perylene model system because these dyes are very attractive for organic electronics due to their high stability and suitable optical and transport properties. ${ }^{24-26}$ Due to the large fluorescence quantum yield of these dyes, effective triplet sensitization can be achieved via TEET. ${ }^{7}{ }^{27}$ Moreover, recently enhanced intersystem crossing yields populating triplet states have been reported in stacked perylene dimers similar to our model, ${ }^{28}$ and a new class of perylene derivatives with intrinsic enhanced triplet yields have also been reported. ${ }^{29}$

In order to unravel the effect of the solvent on TEET interactions, we combine classical molecular dynamics simulations of the dimer in water, chloroform and benzene with quantum chemical calculation of the systems, including both the chromophore and solvent molecules. Electronic couplings are estimated using the Fragment Excitation Difference (FED) scheme ${ }^{30}$ applied to the data of CIS (configuration interaction of single excitations) calculations of the systems. We also estimate electronic couplings for hole transfer (HT) and excess electron transfer (EET) in the systems using the Fragment Charge Difference (FCD) ${ }^{31}$ scheme. Comparison of the data allows us to consider whether TEET decay constants may be derived from the corresponding HT and EET parameters. Our results indicate that solvent molecules located between the chromophores affect strongly the TEET coupling, while the effect of solvent molecules outside the dimer is rather small. Overall, the solvent largely boosts the TEET process reducing the decay constant $\beta$ from 5.1 $\AA^{-1}$ in vacuum to 3.4-3.8 $\AA^{-1}$, depending on its nature. Moreover, our results indicate that the distance dependence of TEET can be reasonably well approximated as the sum of HT and EET decay constants, $\beta_{\mathrm{TEET}} \approx \beta_{\mathrm{EET}}$ $+\beta_{\mathrm{HT}}$. 


\section{Methods}

\subsection{FCD calculation of the HT and EET couplings}

The FCD method ${ }^{31}$ (ref. 31) was employed to calculate the coupling for HT and EET between PDI units in the dimer

$$
V_{D A}=\frac{\left(E_{2}-E_{1}\right)\left|\Delta q_{12}\right|}{\sqrt{\left(\Delta q_{1}-\Delta q_{2}\right)^{2}+4 \Delta q_{12}^{2}}}
$$

For HT, the adiabatic splitting $E_{2}-E_{1}$ can be estimated through the one-electron energies of the highest occupied molecular orbitals (HOMOs) of neutral systems; for EET, the gap is expressed through the difference of orbital energies of lowest unoccupied MOs (LUMOs). In Eq. (2), $\Delta q_{1}$ and $\Delta q_{2}$ are the difference of hole (or excess electron) charges on the donor and acceptor sites in the adiabatic states of interest, $\Delta q_{12}$ is the corresponding off-diagonal term. The calculation of these quantities as well as underlying approximations are described in detail in Ref. ${ }^{31}$ and ${ }^{32}$.

\subsection{FED calculation of the TEET coupling}

Within a two state model, the TEET electronic coupling for a symmetric system (with identical donor and acceptor) may be estimated as a half of the excited state splitting $\triangle{ }^{33}$ However, the half-splitting scheme provides only an upper limit of the coupling when the structure deviates from the symmetric geometry. Recently, Hsu et al. introduced the Fragment Excitation Difference (FED) scheme to estimate the coupling for excitation energy transfer. ${ }^{30}$ The FED approach is an elegant extension of FCD. ${ }^{31}$ Because electronic excitation can be viewed as the creation of an electron-hole pair, the excitation electron density can be presented as a sum of the attachment and detachment electron densities. ${ }^{34}$ The TEET coupling is given by

$$
|V|=\frac{\Delta \cdot\left|x_{m n}\right|}{\sqrt{\left(x_{m m}-x_{n n}\right)^{2}+4 \cdot x_{m n}^{2}}}
$$

where $\Delta$ is the energy difference of excited states $m$ and $n$, and the matrix elements $x_{m n}$ are expressed through the excited-state densities:

$$
x_{m n}=\int_{r \in D} \rho_{e x}^{m n}(r) d r-\int_{r \in A} \rho_{e x}^{m n}(r) d r
$$


$\rho_{e x}$ is calculated as the sum of the attachment and detachment densities. The FED scheme can be used in combination with various quantum chemical methods. In the current study, we employ FED together with the nonempirical CIS method.

\subsection{Computational details}

\section{Molecular dynamics simulations}

The stacked structures of the perylene diimide (PDI) dimer, at an interchromophoric $\mathrm{R}=7.0 \AA$ separation, were taken from our previous work. ${ }^{35} \mathrm{MD}$ simulations at $\mathrm{R}=7.0 \AA$ and $\mathrm{R}=10.5 \AA$ in water, chloroform and benzene solutions were carried out, in which the structure of the PDI dimer was kept frozen. The solvated systems were built by embedding the PDI dimer in water, chloroform and benzene boxes (buffer zone of $20 \AA$ ) using the Leap module of the Amber9 suite of programs. ${ }^{36}$ Water and chloroform solvents were described using the TIP $3 \mathrm{P}^{37}$ water and the Amber chloroform ${ }^{38}$ parameters, whereas the $\mathrm{GAFF}^{39}$ force field was employed to describe PDI and benzene solvent molecules. The systems were first thermalized by running a 50-ps MD simulation at constant volume to increase the temperature from $100 \mathrm{~K}$ to $298 \mathrm{~K}$. Subsequently, a 50-ps equilibration at constant pressure (1 atm) and temperature (298 $\mathrm{K})$ was carried out using standard coupling schemes in order to reach appropriate densities of the liquids. Finally, the simulation was extended for $2 \mathrm{~ns}$ for production purposes. All runs were performed with Amber $9^{36}$ using an integration time step of $1 \mathrm{fs,}$ periodic boundary conditions, the Particle Mesh Ewald approach to deal with longrange electrostatics, and a nonbonded cutoff equal to $10 \AA$. For pressure regulation, an isothermal compressibility of $44.6,^{36} 108.6^{38}$ and $96.6 \mathrm{TPa}^{-1}{ }^{40}$ was used for water, chloroform and benzene, respectively.

\section{Electronic coupling calculations}

All systems were computed at the CIS (configuration interaction of single excitations) level with the $6-31+\mathrm{G}(\mathrm{d})$ basis set. Based on these data, we derived the TEET electronic couplings using by the $\mathrm{FED}^{30}$ method. Calculations of hole transfer (HT) and excess electron transfer (EET) couplings were computed using the FCD method $^{31}$ applied to the results of $\mathrm{HF} / 6-31+\mathrm{G}(\mathrm{d})$ calculations of closed shell neutral systems. In previous work, we showed that this level of theory gives TEET couplings in good agreement with correlated EOM-CCSD calculations, thus providing a good balance between accuracy and computational efficiency. ${ }^{41}$ From each MD trajectory in water, chloroform and benzene, 10 uncorrelated structures were extracted. The snapshots, separated by 100 ps, were taken from the last ns. In order to explore the effect of the intervening medium, the solvent molecules located in the inter- 
chromophoric space were explicitly considered in the quantum chemical calculations, as illustrated in Figure 1, whereas all other solvent molecules were discarded. Ab initio calculations were carried out using the Gaussian09 package. ${ }^{42}$ The TEET, HT and EET couplings were derived by processing the Gaussian outputs with the FCM program. ${ }^{43}$ Finally, additional calculations with a PDI-PDI $R=3.5 \AA$ separation were performed in order to generate starting values for the description of the coupling distance decay.

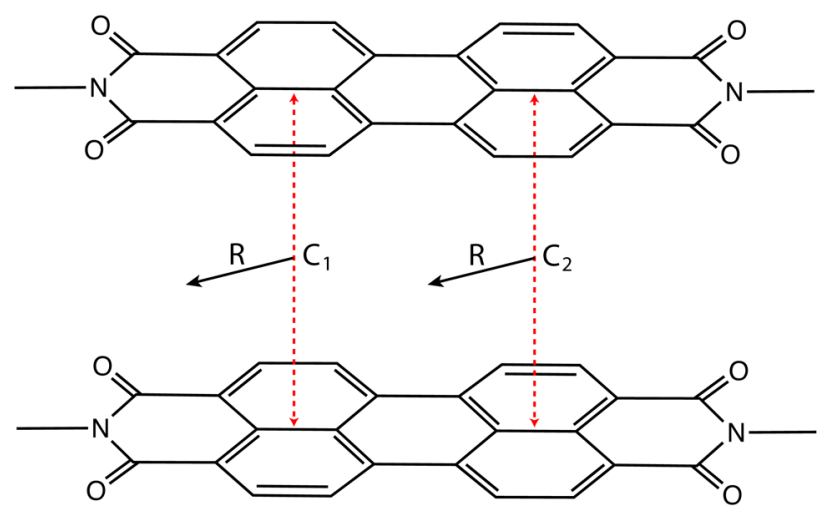

Figure 1. Structure of the perylene diimide dimer studied in this work. In order to explore the effect of the intervening medium, the solvent molecules located closer to either the $\mathrm{C}_{1}$ or the $\mathrm{C}_{2}$ center were explicitly considered in the calculations.

\section{Results and discussion}

\subsection{Inclusion of explicit solvent and conformational sampling}

In this section we analyze the impact of the number of solvent molecules included in the TEET coupling results. Because of the remarkable computational cost associated with the CIS calculations of the solvated systems, this allows us to balance the computational requirements with a satisfactory accuracy of the estimated TEET couplings. Moreover, by computing electronic coupling values by adding solvent molecules in a sequential manner, we are able to dissect the effect of individual solvent molecules, thus illustrating its constructive or destructive interference effect on the coupling values. In addition, TEET couplings are extremely sensitive to conformational disorder. ${ }^{41}$ Thus, we also explore the impact of conformational disorder on the coupling values derived in order to determine the minimal number of structures necessary to obtain converged results.

In Figure 2 we illustrate representative structures of the PDI dimer in water, chloroform and benzene. We recall that the MD simulations were performed by keeping the PDI 
molecules frozen at the separation $\mathrm{R}=7.0$ and $10.5 \AA$. Using a frozen geometry for the PDI dimer in the two simulations allows us to exclude coupling fluctuations due to motions of the PDI units, given that our aim here is to explore the effect of the solvent, and solvent fluctuations, on the distance dependence of coupling values.

In Figure 3 we show the dependence of the coupling on the number of solvent molecules included in the calculations for a representative snapshot extracted from the MD trajectory. At $\mathrm{R}=7.0 \AA$, the convergence of the results is very fast. Satisfactory coupling values in water and chloroform are obtained with just 10 and 4 solvent molecules. Thus the solvent effect on the TEET coupling is mainly due to the solvent molecules located between the chromophores. The trend obtained in benzene is similar. However, in this case some variation is found when passing from 2 to 4 benzenes, and two of such benzenes are not strictly located in the interchromophore region, as illustrated in Figure 2. At the separation of $10.5 \AA$, the convergence of coupling values is significantly slower, indicating a more complex effect of the solvent arrangement. Convergence is achieved at 30 waters, 9 chloroform and 6 benzenes molecules. Given these results, in order to explore the impact of conformational flexibility, as well as in all results on next section, we limit the number of solvent molecules and perform calculations including 5 benzene, 7 chloroform, and 25 water molecules for the dimer with $\mathrm{R}=7.0 \AA$; and 7,9 and 35 solvent molecules when calculating the dimer with $\mathrm{R}=10.5 \AA$. The fact that only intervening solvent molecules modulate the coupling between PDIs can be explained from the orbital overlap-dependence of TEET interactions. ${ }^{12-13}$ This is in sharp contrast with solvent effects on singlet-singlet energy transfer, where the long-range nature of Coulomb interactions characterizing singlet migration means that large solvation shells need to be included in order to recover the screening effect induced by the solvent. ${ }^{35}$

In Figure 4 we show the dependence of the mean coupling value, $\left\langle\mathrm{V}^{2}>\right.$, on the number of structures extracted from the MD trajectory. The values in water show little dependence on the number of structures considered. This can be explained by the fact that many water molecules are mediating the coupling. Thus, the enhancement/decrease on the coupling arising from changes on the relative orientation of particular waters can be counterbalanced by the fluctuations of the other waters. In contrast, in chloroform and benzene solutions the solvent-mediated coupling arises from a few number of solvent molecules, thus one can expect that fluctuations on the relative orientation between these solvent molecules and the PDI units has a larger impact on the coupling values. In other words, the solvent-mediated coupling depends on the relative orientation of a few solvent molecules. This is indeed the case, especially for benzene at $\mathrm{R}=7 \AA$, where the coupling is mainly mediated by two benzenes stacked in between the perylene units. Nevertheless, in all cases the $\left\langle\mathrm{V}^{2}\right\rangle$ values averaged over 10 structures appear to be well converged. We note that TEET as well as HT and EET couplings are exquisitely sensitive to the particular solvent conformation and small variations in the orientation of PDI units and solvent molecules can lead to remarkable changes of the matrix elements. We find that TEET couplings may change by up to two orders of 
magnitude by passing between different configurations of solvent molecules. Thus to properly describe the solvent effects on the coupling, conformational disorder of solvent molecules must be accounted for (see S.I.). A model based on the treatment of a single solute-solvent structure would not give a realistic description of this effect. In the Supporting Information we provide the structures with the maximum and minimum TEET couplings found in each solvent. The matrix element is controlled by orbital overlap of intervening solvent molecules, so it is very difficult to identify structural features that induce strong electronic couplings (see results for water and chloroform presented by Figures S1 and S2 in the Supporting Information). At the chromophore separation of $10.5 \AA$ in benzene (see Figure S3), better stacking between the intervening benzene molecules and the PDI units increases the orbital overlap, and consequently leads to a large value of the TEET couplings.

\subsection{Solvent effects on the distance-dependence of TEET, HT and EET}

In this section we aim at estimating how an intervening solvent modifies the distancedependence of TEET rates. Due to the exponential distance-dependence of TEET rates, this effect can be quantified through a $\beta$ distance decay parameter:

$$
k_{T E E T}=k_{0} e^{-\beta_{T E E T} R_{D A}}
$$

This expression derives from the exponential attenuation of TEET couplings. In the weak coupling regime, TEET rates follow Fermi's golden rule, thus being proportional to the square of the electronic coupling, $k_{T E E T} \propto V_{T E E T}^{2}$. Thus, the electronic coupling can be related to the $\beta$ distance decay parameter through the following equation:

$$
V_{T E E T}=V_{0} e^{-\frac{\beta_{T E E T}}{2} R_{D A}}
$$

By using the average coupling values reported in Figure 4 and Table 1, we can thus estimate the $\beta$ decay parameter in vacuum and in water, chloroform and benzene solutions, by linear regression of the expression $\ln \left(V_{T E E T}^{2}\right)=\ln \left(V_{0}^{2}\right)-\beta_{\text {TEET }} R_{D A}$. The corresponding results are shown in Figure 5. In all cases, the fit give correlation coefficients $r>0.95$, thus confirming the exponential attenuation of the coupling values in our calculations. As expected, the fastest attenuation of the TEET coupling is found in vacuum, with $\beta_{\mathrm{TEET}}=5.14 \AA^{-1}$. Then, progressively attenuated decays are obtained in water, chloroform and benzene, with $\beta_{\text {TEET }}$ values equal to $3.77,3.61$ and $3.44 \AA^{-1}$, respectively. The value in vacuum agrees well with $\beta_{\text {TEET }}$ parameters calculated recently by $\mathrm{Hsu}$ and co-workers in the range 5.0-5.8 $\AA^{-1}$ for a series of stacked all-trans 
polyacetylenes and polycyclic aromatic hydrocarbons using the similar Fragment Spin Difference (FSD) method. ${ }^{17}$

The solvent dependence of the $\beta_{\text {TEET }}$ values can be qualitatively explained based on the superexchange nature of the solvent effect. According to the superexchange model introduced by McConnell to model electron transfer reactions, the bridge-mediated donor-acceptor interaction can be described by the following equation: ${ }^{14-15}$

$$
V_{D A}=\frac{V_{D B} V_{B A}}{\Delta E_{D B}}\left(\frac{V_{B B}}{\Delta E_{D B}}\right)^{n-1}
$$

where $V_{D B}, V_{B A}$ and $V_{B B}$ are coupling matrix elements between donor-bridge, bridgeacceptor, and bridge-bridge states, whereas $\Delta E_{D B}$ is the energy difference between the donor and the bridge. This equation assumes that $n$ identical intervening molecules mediate the coupling. In our case, the situation is more complex because the donor and acceptor are coupled differently to several bridging solvent molecules mediating the coupling. Another aspect is the dependence of the coupling on the energy gap between the triplet states of donor and bridge (solvent). According to our calculations, the $\Delta E_{D B}$ values are 7.1, 5.3 and $2.1 \mathrm{eV}$ in water, chloroform and benzene, thus suggesting a decrease of $\beta_{\text {TEET }}$ by passing from water to chloroform and benzene.

As described in the introduction, a common approximation relates the TEET coupling with HT and EET couplings as $\left|V_{T E E T}\right| \approx C\left|V_{E E T}\right|\left|V_{H T}\right| \cdot{ }^{12-13,15}$ Accordingly, the TEET attenuation factor can be expressed as the sum of HT and EET factors, $\beta_{\text {TEET }} \approx \beta_{\text {EET }+}$ $\beta_{\mathrm{HT}}$. When $\beta_{\mathrm{HT}} \approx \beta_{\mathrm{EET}}$, the decay parameter for TEET is roughly twice that for EET (or $\mathrm{HT}), \beta_{\mathrm{TEET}} / \beta_{\mathrm{EET}} \approx 2$. In order to critically assess these assumptions, in Table 1 we report squared coupling values for HT and EET reactions in the PDI dimer. In Figure 5 we show the distance decay of the couplings along with the corresponding $\beta$ parameters. Interestingly, HT decays show a marked dependence on the solvent, whereas the EET parameters remain quite similar in the different environments. This can be qualitatively explained as follows. The EET coupling is determined by the overlap of the LUMOs of the neutral chromophores, whereas the HT coupling depends on overlap of the corresponding HOMOs. Because HOMOs are less diffuse than LUMOs, the decay of the HT coupling in vacuum should be much more pronounced than that of EET. Therefore, the solvent-mediated effect is expected to be stronger for HT. As TEET can be seen as a simultaneous HT and EET process, its solvent-dependence is expected in between those of HT and EET. Indeed, our calculations suggest that the impact of the solvent on the decay constants follows the trend HT $>$ TEET $>$ EET. As expected, the $\beta$ constants for HT and EET are roughly half as large as $\beta_{\text {TEET }}$. However, because of the different solvent-dependence of TEET, HT and EET, significant deviations from $\beta_{\mathrm{TEET}} / \beta_{\mathrm{EET}}=2$ and $\beta_{\mathrm{TEET}} / \beta_{\mathrm{HT}}=2$ are observed in some cases (Table 2 ). 
Note that the ratios $\beta_{\mathrm{TEET}} / \beta_{\mathrm{EET}}$ and $\beta_{\mathrm{TEET}} / \beta_{\mathrm{HT}}$ show opposite trends when passing from vacuum to water, chloroform and benzene. Because of that the relation $\beta_{\mathrm{TEET}} \approx \beta_{\mathrm{EET}}+$ $\beta_{\mathrm{HT}}$ approximates reasonably well the solvent-dependence of the decay constants $\beta_{\text {TEET }}$ (see Table 2), given that deviations from the $\beta_{\mathrm{TEET}} / \beta_{\mathrm{EET}}=2$ and $\beta_{\mathrm{TEET}} / \beta_{\mathrm{HT}}=2$ ratios are counterbalanced. This holds good for the PDI dimer in benzene and vacuum, whereas in chloroform and water $\beta_{\mathrm{EET}}+\beta_{\mathrm{HT}}$ overestimates $\beta_{\mathrm{TEET}}$ by $\sim 15 \%$. This difference could partly arise from the different quantum chemical approaches used to compute the couplings (TEET is treated by CIS while and EET and HT within the one-electron approximation). Unfortunately, more accurate quantum mechanical methods, e.g. MSCASPT $2{ }^{44}$ cannot be applied to the systems considered in this study.

In conclusion, our results indicate that the relation $\beta_{\mathrm{TEET}} \approx \beta_{\mathrm{EET}}+\beta_{\mathrm{HT}}$ is reasonably valid, while the relation $\beta_{\mathrm{HT}} \approx \beta_{\mathrm{EET}}$ does not always hold due to the different solvent-dependence of the HT and EET reactions. In this case, the EET data provide better estimates of the TEET decay constant $\left(\beta_{\mathrm{TEET}}=2 \beta_{\mathrm{EET}}\right)$ than the HT data. 


\section{Water}
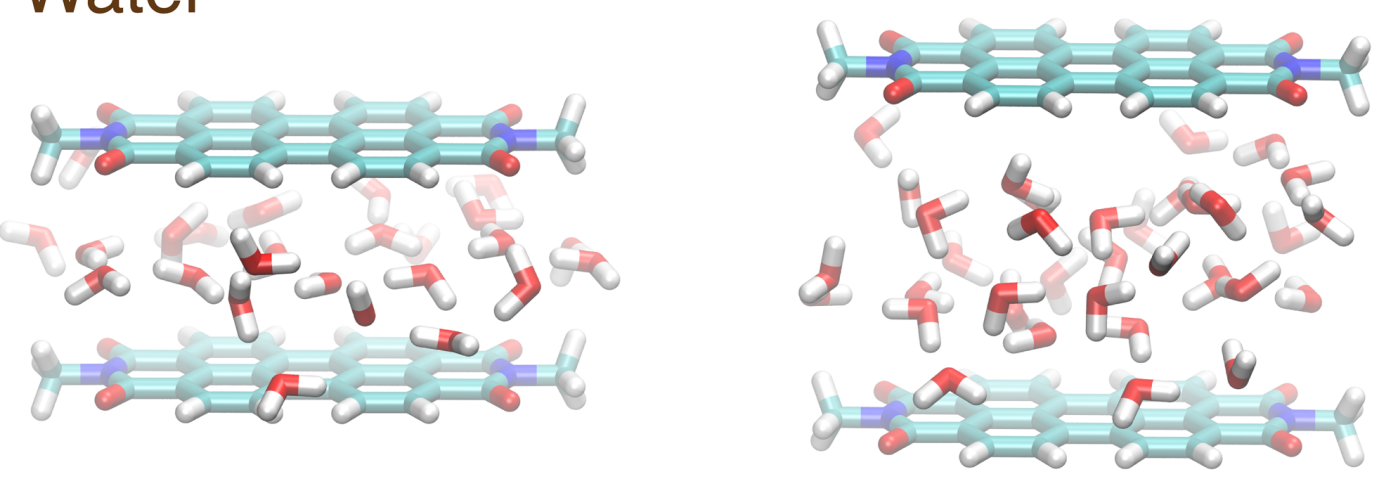

\section{Chloroform}
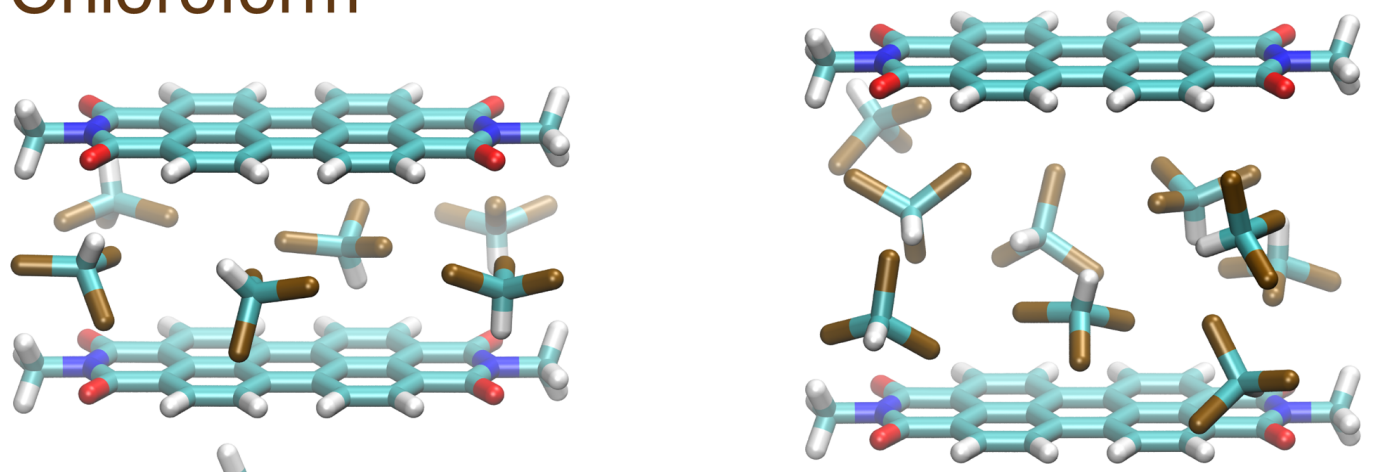

\section{Benzene}
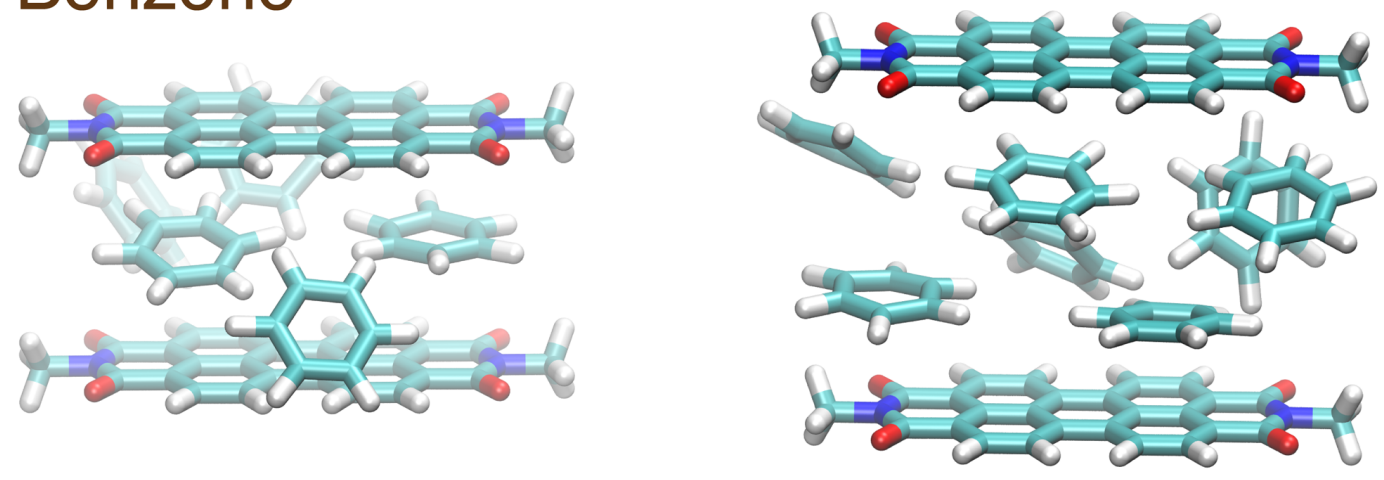

Figure 2. Structure of representative snapshots of the PDI dimer with separations $R=7.0$ and $10.5 \AA$ in water, chloroform and benzene solutions. 
a)

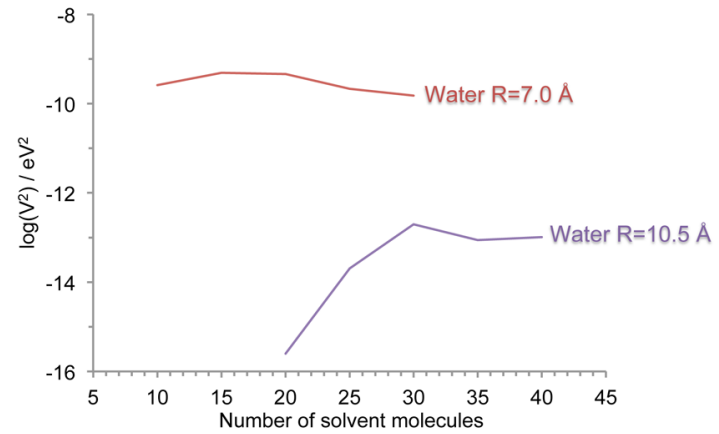

b)

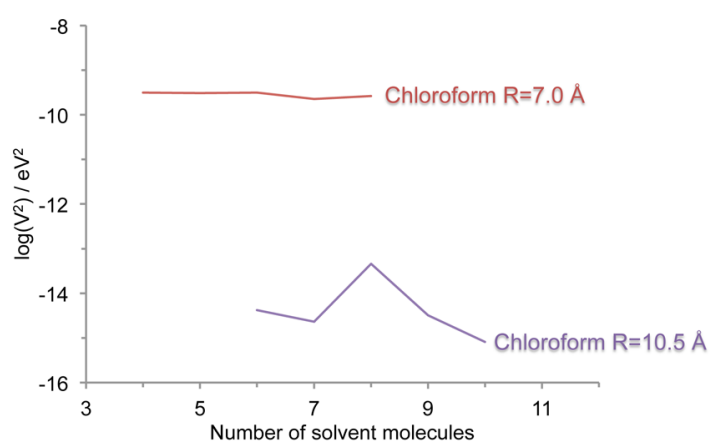

c)

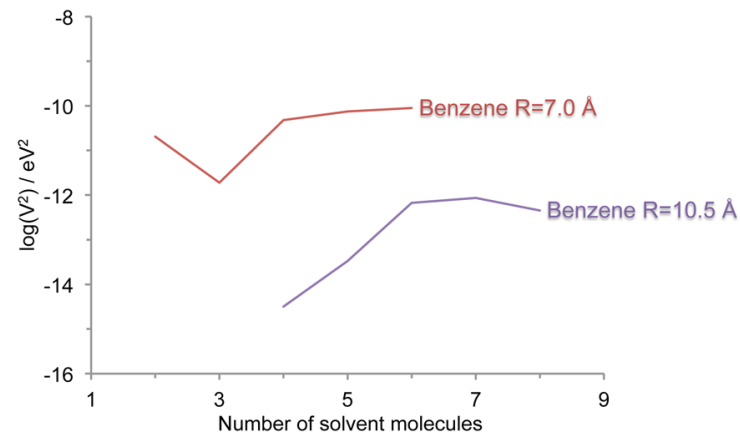

Figure 3. Dependence of TEET couplings at $\mathrm{R}=7.0$ and $10.5 \AA$ inter-chromophoric separations on the number of solvent molecules included in the model. The data in water, chloroform and benzene are computed at the CIS/6-31+G(d) level of theory 
a)

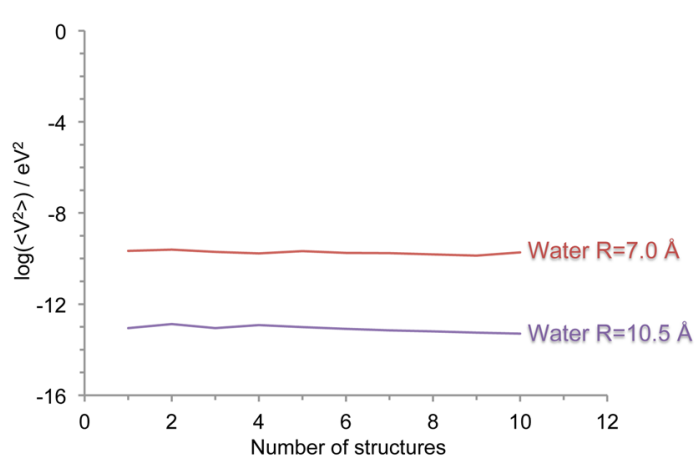

b)

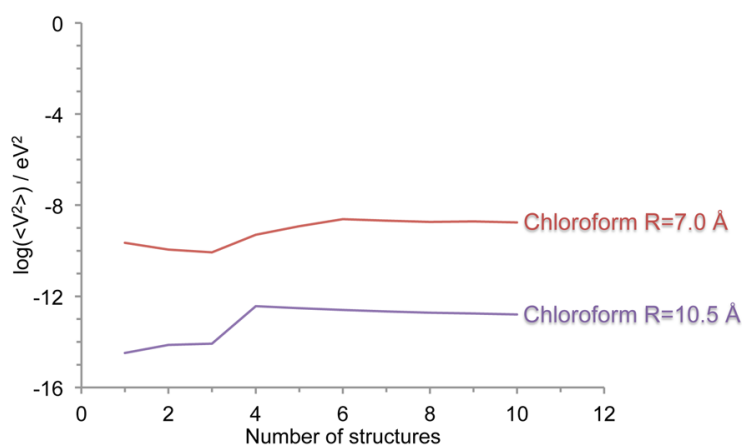

c)

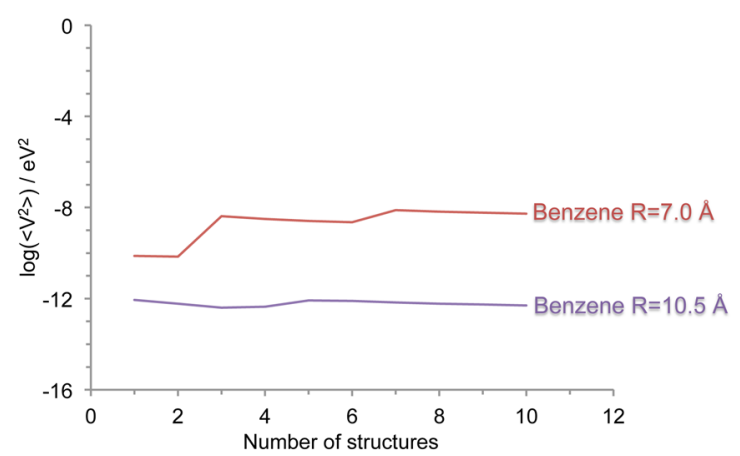

Figure 4. Dependence of TEET couplings at $\mathrm{R}=7.0$ and $10.5 \AA$ inter-chromophoric separations on the number of structures sampled from the classical MD simulation in a) water, b) chloroform and c) benzene. 
a)

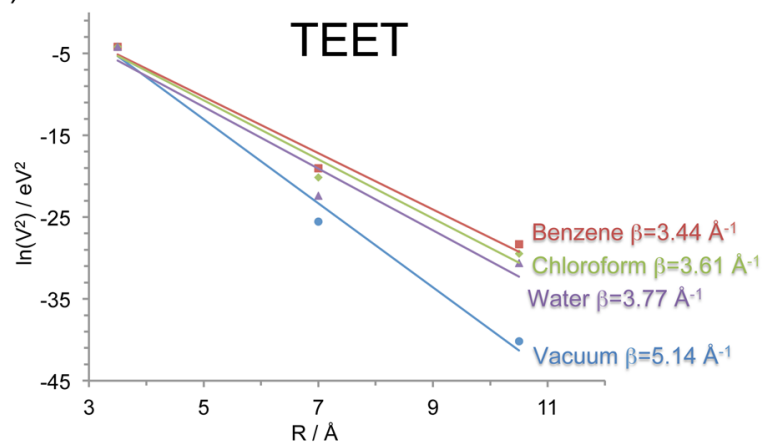

b)

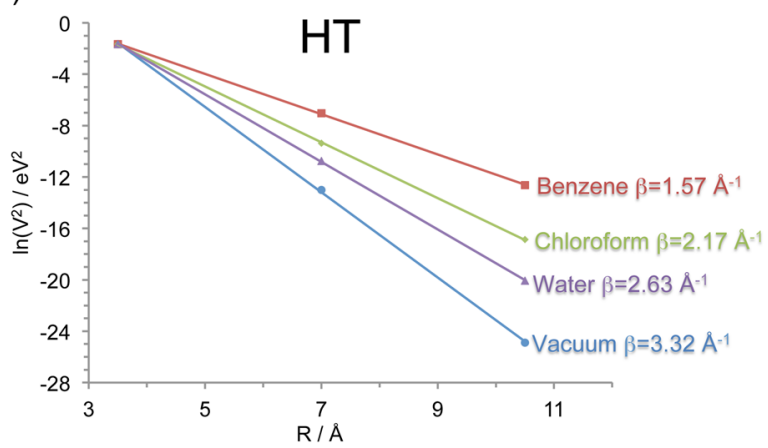

c)

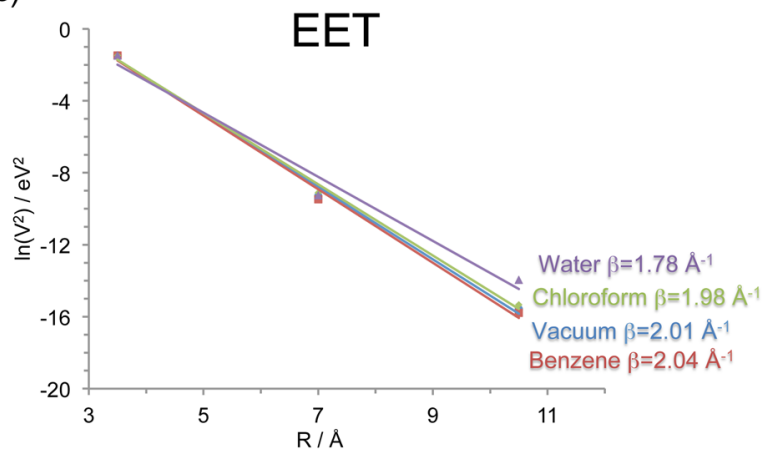

Figure 5. Comparison of the $\beta$ distance decay parameter for triplet-triplet energy transfer (TEET), hole transfer (HT) and excess electron transfer (EET) in vacuum, and in water, chloroform and benzene solutions. 
Table 1. Average squared couplings corresponding to HT, EET and TEET reactions at $\mathrm{R}=3.5,7.0$ and $10.5 \AA$ inter-chromophoric separations in different solvents.

\begin{tabular}{|c|c|c|c|}
\hline$<\mathrm{V}^{2}>/ \mathrm{eV}^{2}$ & $\mathrm{R}=3.5 \AA$ & $\mathrm{R}=7.0 \AA$ & $\mathrm{R}=10.5 \AA$ \\
\hline \multicolumn{4}{|l|}{ Vacuum } \\
\hline HT & $1.89 \cdot 10^{-1}$ & $2.25 \cdot 10^{-6}$ & $1.55 \cdot 10^{-11}$ \\
\hline EET & $2.28 \cdot 10^{-1}$ & $9.22 \cdot 10^{-5}$ & $1.71 \cdot 10^{-7}$ \\
\hline TEET & $1.49 \cdot 10^{-2}$ & $7.83 \cdot 10^{-12}$ & $3.56 \cdot 10^{-18}$ \\
\hline \multicolumn{4}{|l|}{ Water } \\
\hline HT & $1.89 \cdot 10^{-1}$ & $2.15 \cdot 10^{-5}$ & $1.94 \cdot 10^{-9}$ \\
\hline EET & $2.28 \cdot 10^{-1}$ & $9.60 \cdot 10^{-5}$ & $8.76 \cdot 10^{-7}$ \\
\hline TEET & $1.49 \cdot 10^{-2}$ & $1.86 \cdot 10^{-10}$ & $5.05 \cdot 10^{-14}$ \\
\hline \multicolumn{4}{|l|}{ Chloroform } \\
\hline HT & $1.89 \cdot 10^{-1}$ & $8.56 \cdot 10^{-5}$ & $4.71 \cdot 10^{-8}$ \\
\hline EET & $2.28 \cdot 10^{-1}$ & $1.10 \cdot 10^{-4}$ & $2.20 \cdot 10^{-7}$ \\
\hline TEET & $1.49 \cdot 10^{-2}$ & $1.75 \cdot 10^{-9}$ & $1.58 \cdot 10^{-13}$ \\
\hline \multicolumn{4}{|l|}{ Benzene } \\
\hline HT & $1.89 \cdot 10^{-1}$ & $8.81 \cdot 10^{-4}$ & $3.26 \cdot 10^{-6}$ \\
\hline EET & $2.28 \cdot 10^{-1}$ & $7.64 \cdot 10^{-5}$ & $1.39 \cdot 10^{-7}$ \\
\hline TEET & $1.49 \cdot 10^{-2}$ & $5.36 \cdot 10^{-9}$ & $5.03 \cdot 10^{-13}$ \\
\hline
\end{tabular}


Table 2. Exponential decay parameters characterizing HT, EET and TEET reactions in different solvents.

\begin{tabular}{lcccccc}
\hline$\beta / \AA^{-1}$ & $\beta_{\mathrm{HT}}$ & $\beta_{\mathrm{EET}}$ & $\beta_{\mathrm{HT}}+\beta_{\mathrm{EET}}$ & $\beta_{\mathrm{TEET}}$ & $\beta_{\mathrm{TEET}} / \beta_{\mathrm{HT}}$ & $\beta_{\mathrm{TEET}} / \beta_{\mathrm{EET}}$ \\
\hline Benzene & 1.57 & 2.04 & 3.61 & 3.44 & 2.19 & 1.69 \\
Chloroform & 2.17 & 1.98 & 4.15 & 3.61 & 1.66 & 1.82 \\
Water & 2.63 & 1.78 & 4.41 & 3.77 & 1.43 & 2.12 \\
Vacuum & 3.32 & 2.01 & 5.33 & 5.14 & 1.55 & 2.56 \\
\end{tabular}

\section{Conclusions}

In this study, we calculated effective electronic couplings for triplet excitation energy transfer as well as for hole and excess electron transfer between $\pi$ stacked PDI molecules in water and two quite different organic solvents (chloroform and benzene). MD simulation of the systems were performed to account for the effects of conformational motion of solvent molecules. Because the transfer integrals are found to be sensitive to structural fluctuations, the effective couplings were estimated by averaging over selected configurations. The superexchange interaction with solvent molecules has been found to improve significantly electronic communication between the donor and acceptor sites in the considered systems. We have estimated the distance decay parameter $\beta$ for the energy and electron transfer processes in the systems and found that decay parameter for solvent induced TEET can be reasonably well approximated by the sum of $\beta_{\mathrm{EET}}$ and $\beta_{\mathrm{HT}}$. Thus, the relation between the couplings for energy and electron transfer derived by Scholes et al $^{12-13}$ for direct electronic interaction of donor and acceptor may be applied in a more general context.

\section{Acknowledgements}

C.C. acknowledges support from the Spanish Ministry of Economy and Competitivity through the Ramón y Cajal program (Ref. RYC-2011-08918). A.A.V. acknowledges financial support from MICINN (Ministry of Science and Innovation, Spain) and the FEDER fund (European Fund for Regional Development) provided by grants CTQ 2011-26573, UNGI08-4E-003 and UNGI10-4E-801. 


\section{Supporting Information Available}

Figures reporting the structures of the PDI dimer - solvent systems showing the maximum and minimum coupling values in water, chloroform and benzene. This information is available free of charge via the Internet at http://pubs.acs.org.

\section{References}

1. Andrews, D. L.; Curutchet, C.; Scholes, G. D. Laser Photonics Rev. 2011, 5, 114-123.

2. Holt, N. E.; Fleming, G. R.; Niyogi, K. K. Biochemistry 2004, 43, 8281-8289.

3. Kohler, A.; Bassler, H. Mat. Sci. Eng. R 2009, 66, 71-109.

4. Kohler, A.; Bassler, H. J. Mater. Chem. 2011, 21, 4003-4011.

5. Vura-Weis, J.; Abdelwahed, S. H.; Shukla, R.; Rathore, R.; Ratner, M. A.; Wasielewski, M. R. Science 2010, 328, 1547-1550.

6. Shao, Y.; Yang, Y. Adv. Mater. 2005, 17, 2841-2844.

7. Wu, W. H.; Guo, H. M.; Wu, W. T.; Ji, S. M.; Zhao, J. Z. J. Org. Chem. 2011, 76, 7056-7064.

8. $\quad$ Keivanidis, P. E.; Laquai, F.; Robertson, J. W. F.; Baluschev, S.; Jacob, J.; Mullen, K.; Wegner, G. J. Phys. Chem. Lett. 2011, 2, 1893-1899.

9. Closs, G. L.; Piotrowiak, P.; Macinnis, J. M.; Fleming, G. R. J. Am. Chem. Soc. 1988, $110,2652-2653$.

10. Closs, G. L.; Johnson, M. D.; Miller, J. R.; Piotrowiak, P. J. Am. Chem. Soc. 1989, 111, 3751-3753.

11. Dexter, D. L. J. Chem. Phys. 1953, 21, 836-850.

12. Harcourt, R. D.; Scholes, G. D.; Ghiggino, K. P. J. Chem. Phys. 1994, 101, 1052110525 .

13. Scholes, G. D.; Harcourt, R. D.; Ghiggino, K. P. J. Chem. Phys. 1995, 102, 9574-9581.

14. McConnell, H. J. Chem. Phys. 1961, 35, 508-515.

15. Albinsson, B.; Martensson, J. Phys. Chem. Chem. Phys. 2010, 12, 7338-7351.

16. You, Z. Q.; Hsu, C. P.; Fleming, G. R. J. Chem. Phys. 2006, 124, 044506.

17. You, Z.-Q.; Hsu, C.-P. J. Chem. Phys. 2010, 133, 074105.

18. Yeganeh, S.; Van Voorhis, T. J. Phys. Chem. C 2010, 114, 20756-20763.

19. Subotnik, J. E.; Vura-Weis, J.; Sodt, A. J.; Ratner, M. A. J. Phys. Chem. A 2010, 114, $8665-8675$.

20. Cave, R. J.; Newton, M. D.; Kumar, K.; Zimmt, M. B. J. Phys. Chem. 1995, 99, 1750117504.

21. Kumar, K.; Kurnikov, I. V.; Beratan, D. N.; Waldeck, D. H.; Zimmt, M. B. J. Phys. Chem. A 1998, 102, 5529-5541.

22. Troisi, A.; Ratner, M. A.; Zimmt, M. B. J. Am. Chem. Soc. 2004, 126, 2215-2224.

23. Mani, T.; Niedzwiedzki, D. M.; Vinogradov, S. A. J. Phys. Chem. A 2012, 116, 35983610.

24. Ahrens, M. J.; Sinks, L. E.; Rybtchinski, B.; Liu, W. H.; Jones, B. A.; Giaimo, J. M.; Gusev, A. V.; Goshe, A. J.; Tiede, D. M.; Wasielewski, M. R. J. Am. Chem. Soc. 2004, 126, 8284-8294.

25. Zhan, X. W.; Facchetti, A.; Barlow, S.; Marks, T. J.; Ratner, M. A.; Wasielewski, M. R.; Marder, S. R. Adv. Mater. 2011, 23, 268-284.

26. Li, C.; Wonneberger, H. Adv. Mater. 2012, 24, 613-636.

27. Ford, W. E.; Kamat, P. V. J. Phys. Chem. 1987, 91, 6373-6380.

28. Veldman, D.; Chopin, S. M. A.; Meskers, S. C. J.; Groeneveld, M. M.; Williams, R. M.; Janssen, R. A. J. J. Phys. Chem. A 2008, 112, 5846-5857. 
29. Ventura, B.; Langhals, H.; Bock, B.; Flamigni, L. Chem. Commun. 2012, 48, 42264228.

30. Hsu, C. P.; You, Z. Q.; Chen, H. C. H. J. Phys. Chem. C 2008, 112, 1204-1212.

31. Voityuk, A. A.; Rösch, N. J. Chem. Phys. 2002, 117, 5607-5616.

32. Rösch, N.; Voityuk, A. A. Top. Curr. Chem. 2004, 237, 37-72.

33. Scholes, G. D.; Ghiggino, K. P. J. Phys. Chem. 1994, 98, 4580-4590.

34. Head-Gordon, M.; Grana, A. M.; Maurice, D.; White, C. A. J. Phys. Chem. 1995, 99, 14261-14270.

35. Curutchet, C.; Munoz-Losa, A.; Monti, S.; Kongsted, J.; Scholes, G. D.; Mennucci, B. J. Chem. Theory Comput. 2009, 5, 1838-1848.

36. Case, D. A.; Darden, T. A.; Cheatham, I., T.E.; Simmerling, C. L.; Wang, J.; Duke, R. E.; Luo, R.; Merz, K. M.; Pearlman, D. A.; Crowley, M. et al. AMBER 9, University of California: San Francisco, 2006.

37. Jorgensen, W. L.; Chandrasekhar, J.; Madura, J. D.; Impey, R. W.; Klein, M. L. J. Chem. Phys. 1983, 79, 926-935.

38. Fox, T.; Kollman, P. A. J. Phys. Chem. B 1998, 102, 8070-8079.

39. Wang, J. M.; Wolf, R. M.; Caldwell, J. W.; Kollman, P. A.; Case, D. A. J. Comput. Chem. 2004, 25, 1157-1174.

40. Diaz Peña, M.; Tardajos, G. J. Chem. Thermodyn. 1978, 10, 19-24.

41. Curutchet, C.; Voityuk, A. A. Angew. Chem. Int. Ed. 2011, 50, 1820-1822.

42. Frisch, M. J.; Trucks, G. W.; Schlegel, H. B.; Scuseria, G. E.; Robb, M. A.; Cheeseman, J. R.; Scalmani, G.; Barone, V.; Mennucci, B.; Petersson, G. A. et al. Gaussian 09, Revision A.2, Gaussian, Inc.: Wallingford CT, 2009.

43. Voityuk, A. A. FCM, Program for Electronic Coupling Calculations, Version 6, 2010.

44. Blancafort, L.; Voityuk, A. A. Journal of Physical Chemistry A 2007, 111, 4714-4719. 
TOC GRAPHIC

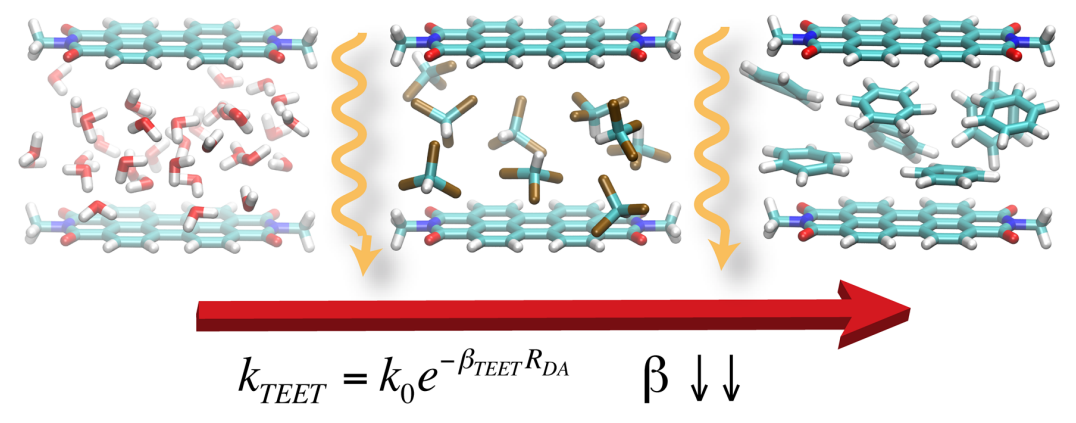

